\title{
Human gut microbiota transferred to germ-free NOD mice modulate the progression towards type 1 diabetes regardless of the pace of beta cell function loss in the donor
}

\author{
Vit Neuman ${ }^{1}$ (D) Ondrej Cinek ${ }^{1}$ - David P. Funda ${ }^{2} \cdot$ Tomas Hudcovic $^{2} \cdot$ Jaroslav Golias $^{2} \cdot$ Lenka Kramna $^{1}$ • \\ Lenka Petruzelkova $^{1} \cdot$ Stepanka Pruhova $^{1} \cdot$ Zdenek Sumnik $^{1}$
}

Received: 5 October 2018 / Accepted: 19 March 2019 /Published online: 25 April 2019

(C) Springer-Verlag GmbH Germany, part of Springer Nature 2019

\begin{abstract}
Aims/hypothesis This study aimed to assess the ability of human gut microbiota to delay the onset of type 1 diabetes when transferred into germ-free NOD mice.

Methods Two children with rapid and three children with slow beta cell function loss (as assessed by C-peptide AUC change in the mixed-meal tolerance tests performed 1 and 12 months after type 1 diabetes onset), participating in an ongoing trial with gluten-free diet, donated faeces, which were transferred into germ-free NOD mice. The mice were subsequently followed for diabetes incidence.

Results The bacterial profiles of bacteriome-humanised mice had significantly $\left(p<10^{-5}\right)$ lower alpha diversity than the donor material, with marked shifts in ratios between the main phyla. Diabetes onset was significantly delayed in all bacteriomehumanised colonies vs germ-free NOD mice, but the pace of beta cell loss was not transferable to the mouse model.

Conclusions/interpretation Germ-free NOD mice colonised with human gut microbiome are able to adopt a large proportion of transferred bacterial content, although the ratios of main phyla are reproduced only suboptimally. The recipient mice did not replicate the phenotype of the stool donor in relation to the pace towards type 1 diabetes.
\end{abstract}

Trial registration ClinicalTrials.gov NCT02867436

Keywords Germ-free NOD mice · Human gut microbiome transfer · Type 1 diabetes

\begin{abstract}
Abbreviations
ILN Inguinal lymph node

MLN Mesenteric lymph node

MMTT Mixed-meal tolerance test

PLN Pancreatic lymph node

Treg T-regulatory cell
\end{abstract}

Electronic supplementary material The online version of this article (https://doi.org/10.1007/s00125-019-4869-2) contains peer-reviewed but unedited supplementary material, which is available to authorised users.

Vit Neuman

Vit.Neuman@fnmotol.cz

1 Department of Pediatrics, 2nd Faculty of Medicine, Charles University and University Hospital Motol, V Úvalu 84, CZ-15006, Prague 5, Czech Republic

2 Institute of Microbiology of the Czech Academy of Sciences, v.v.i., Prague and Novy Hradek, Czech Republic

\section{Introduction}

The role of the gut bacteriome in type 1 diabetes pathogenesis remains elusive [1]. A number of studies were published in the last few years with the development of massive parallel bacterial 16S ribosomal DNA sequencing. None of these provided a definite answer, yet they repeatedly found distortions in the abundance of candidate species among the Bacteroides genus before the initiation of beta cell autoimmunity [2] or before disease onset [3]. Various factors [4] have been associated with the pace of residual beta cell capacity decrease after the disease onset but evidence is lacking for the involvement of gut microbiome.

Gnotobiotic animal models have been used as gut microbiota recipients to help decipher its impact on conditions including type 1 diabetes [5]. We aimed to investigate whether the different ability to conserve the beta cell capacity in children 


\section{Research in context}

\section{What is already known about this subject?}

- The gut microbiome might play a role in the development of type 1 diabetes, yet so far no single causative microorganism has been identified

- There are no conclusive data on the effect of transfer of human gut microbiota on diabetes incidence in germ-free NOD mice

\section{What is the key question?}

- Is the donor phenotype in relation to beta cell function loss transferrable to germ-free NOD mice through gut microbiota?

\section{What are the new findings?}

- Human gut microbiota transfer decreases diabetes incidence in germ-free NOD mouse recipients, irrespective of the pace of beta cell loss in the human donor

- Massive shifts were observed in all bacterial genera yet no conclusive pattern in relation to diabetes incidence was observed

How might this impact on clinical practice in the foreseeable future?

- The human phenotype of beta cell capacity preservation is not transferable through the gut microbiota to NOD mice, the most widely used model of human type 1 diabetes

1 year after type 1 diabetes onset can be passed on to germfree NOD mice through gut microbiota transfer.

\section{Methods}

Gut microbiome donors Five children aged 9-16 years (mean age 13.5 , SD 2.9 years) with type 1 diabetes were chosen based on the results of their AUC of C-peptide in standard mixed-meal tolerance tests (MMTT) performed 1 and 12 months after type 1 diabetes onset (Table 1). The MMTTs were performed within the protocol of an ongoing intervention trial to slow the progression of beta cell loss by instituting a gluten-free diet (ClinicalTrials.gov registration no. NCT02867436). Written informed consent was signed by the caregivers of all participants and the study protocol was approved by the institutional Ethics Committee.

Three of them upheld a strict gluten-free diet while two had a standard diet regimen. Two of the donors (aged 8 and 15 years) belonged to the tertile of participants who lost their beta cell capacity rapidly ( $>75 \%$ function loss over 1 year) whereas three of them (aged 11, 14 and 14 years) were among the tertile of participants with very slow progression of beta cell loss ( $<28 \%$ function loss over 1 year). At 12 months after the type 1 diabetes onset the participants were asked to donate a fresh stool sample, which was immediately processed within $2 \mathrm{~h}$ [6] and kept frozen with buffered glycerol at $-80^{\circ} \mathrm{C}$ until the transfer. The transferred mixture was analysed before and immediately after the procedure to verify that the composition remained stable.
Germ-free NOD mouse recipients Five colonies of 3-4-weekold female germ-free NOD mice (15-20 mice per colony) originally purchased from Taconic (Albany, NY, USA) were used as recipients of the processed material. Oral gavage, enema and direct administration on the skin were used to inoculate each mouse. The inoculum size per mouse ranged from $1.7 \times 10^{8}$ to $1.9 \times 10^{10}$ (mean $6.1 \times 10^{9}$ ) bacteria, as assayed by quantitative pan-bacterial real-time PCR. A control colony of 20 NOD mice who remained germ-free was also observed. The sterility of germ-free NOD mice was verified by culture and pan-bacterial PCR every 14 days prior to the transfer. Mice from each colony were co-housed in sterile containers and fed standard $50 \mathrm{kGy}$-irradiated sterile pellets and autoclaved water ad libitum. Mice were followed for diabetes incidence by weekly measurements of blood glucose levels from a drop of blood obtained from a facial prick. Mice were considered diabetic once their blood glucose exceeded $12 \mathrm{mmol} / \mathrm{l}$. All animal experiments were conducted in concordance with the Guide for the Care and Use of Laboratory Animals (2011).

Stool bacteriome analysis Stool samples were collected from each mouse individually at 14-day intervals and at least once after the diabetes diagnosis. Mouse stools were kept at $-80^{\circ} \mathrm{C}$ until the DNA was extracted using the PowerSoil DNA isolation kit (Qiagen, Hilden, Germany). Microbiome profiles were determined by next-generation sequencing of the V4 variable region of $16 \mathrm{~S}$ [7] and processed in a bioinformatic pipeline as described previously using the Mothur, Qiime and Phyloseq tools [8]. The quantities of two species of Bacteroides, whose 
Table 1 Stool donor characteristics

\begin{tabular}{|c|c|c|c|c|c|}
\hline \multirow[t]{2}{*}{ Characteristic } & \multicolumn{5}{|l|}{ Stool sample donor } \\
\hline & Rapid 1 & Rapid 2 & Slow 1 & Slow 2 & Slow 3 \\
\hline \multicolumn{6}{|l|}{ Demographics } \\
\hline Sex & $\mathrm{F}$ & M & M & $\mathrm{F}$ & M \\
\hline Age (years) & 7 & 14 & 13 & 10 & 13 \\
\hline Body weight (kg) & 27 & 81 & 48 & 32 & 56 \\
\hline \multicolumn{6}{|l|}{ T1D onset data } \\
\hline $\mathrm{HbA}_{1 \mathrm{c}}(\mathrm{mmol} / \mathrm{mol})$ & 87 & 123 & 87 & 111 & 99 \\
\hline $\mathrm{HbA}_{1 \mathrm{c}}(\%)$ & 10.1 & 13.4 & 10.1 & 12.3 & 11.2 \\
\hline $\mathrm{pH}$ & 7.27 & 7.43 & N/A & 7.31 & 7.41 \\
\hline $\begin{array}{l}H L A-D Q B 1 \\
D Q A 1 \text { genotype }\end{array}$ & $\begin{array}{l}D Q B 1 * 02 / 03: 02 \\
D Q A 1 * 03 / 05\end{array}$ & $\begin{array}{l}D Q B 1 * 02 / 03: 02 \\
D Q A 1 * 02 / 03\end{array}$ & $\begin{array}{l}D Q B 1 * 02 / 02 \\
D Q A 1 * 05 / 05\end{array}$ & $\begin{array}{l}D Q B 1 * 03: 01 / 06: 01 \\
D Q A 1 * 01 / 03\end{array}$ & $\begin{array}{l}D Q B 1 * 02 / 03: 02 \\
D Q A 1 * 03 / 05\end{array}$ \\
\hline Autoantibodies & $\begin{array}{l}\mathrm{GAD} 5^{+} \\
\mathrm{IA}^{+} \\
\mathrm{ZnT}^{+}\end{array}$ & $\begin{array}{l}\mathrm{GAD}^{+} 5^{+} \\
\mathrm{IAA}^{+} \\
\mathrm{IA2}^{+} \\
\mathrm{ZnT}^{+}\end{array}$ & $\begin{array}{l}\mathrm{GAD} 5^{+} \\
\mathrm{IA}^{+} \\
\mathrm{ZnT}^{+}\end{array}$ & $\begin{array}{l}{\text { GAD } 65^{+}}^{+} \\
\text {IA }^{+}\end{array}$ & $\begin{array}{l}\mathrm{GAD}^{+} 5^{+} \\
\mathrm{ZnT}^{+}\end{array}$ \\
\hline Dietary intervention & Gluten-free diet & Standard diet & Gluten-free diet & Gluten-free diet & Standard diet \\
\hline \multicolumn{6}{|l|}{ Baseline data } \\
\hline $\begin{array}{l}\text { MMTT C-peptide AUC at } \\
1 \text { month }(\mathrm{pmol} / 1 \times \mathrm{min})\end{array}$ & 1270 & 2227 & 2334 & 2350 & 2233 \\
\hline $\mathrm{HbA}_{1 \mathrm{c}}$ at $1 \mathrm{month}(\mathrm{mmol} / \mathrm{mol})$ & 65 & 75 & 62 & 56 & 68 \\
\hline $\mathrm{HbA}_{1 \mathrm{c}}$ at 1 month $(\%)$ & 8.1 & 9.0 & 7.8 & 7.3 & 8.4 \\
\hline $\begin{array}{l}\text { Daily dose of insulin at } 1 \text { month } \\
(\mathrm{U} / \mathrm{kg})\end{array}$ & 0.29 & 1.2 & 0.38 & 0.25 & 0.4 \\
\hline \multicolumn{6}{|l|}{ Progression of T1D } \\
\hline $\begin{array}{l}\text { MMTT C-peptide AUC at } \\
12 \text { months }(\mathrm{pmol} / 1 \times \mathrm{min})\end{array}$ & 300 & 89 & 1840 & 1693 & 2028 \\
\hline $\begin{array}{l}\text { MMTT C-peptide AUC loss } \\
\text { from baseline }(\%)\end{array}$ & 76.4 & 96.0 & 21.2 & 28.0 & 9.2 \\
\hline $\mathrm{HbA}_{1 \mathrm{c}}$ at 12 months $(\mathrm{mmol} / \mathrm{mol})$ & 75 & 70 & 48 & 44 & 49 \\
\hline $\mathrm{HbA}_{1 \mathrm{c}}$ at 12 months $(\%)$ & 9.0 & 8.6 & 6.5 & 6.2 & 6.6 \\
\hline $\begin{array}{l}\text { Daily dose of insulin at } \\
12 \text { months }(\mathrm{U} / \mathrm{kg})\end{array}$ & 0.60 & 1.36 & 0.52 & 0.25 & 0.44 \\
\hline
\end{tabular}

F, female; M, male; T1D, type 1 diabetes.

associations with islet autoimmunity have been previously proposed [2], were assayed using real-time PCR [8].

Insulitis scores and tissue T-regulatory cell analysis Insulitis levels were scored on H\&E-stained sections and graded as follows: 0 , intact islet; 1 , peri-insulitis; 2 , insulitis, $<50 \%$ infiltration; 3 , insulitis, $>50 \%$ infiltration. A minimum of 25 islets per mouse and 4-6 mice per group were counted. The colonies from 'Slow 1' and 'Rapid 1' did not contain enough mice to allow for insulitis scoring and T-regulatory cell (Treg) assessment in prediabetic mice (12-13 weeks old).

Cell suspensions from spleen, mesenteric lymph nodes (MLNs), pancreatic lymph nodes (PLN) and inguinal lymph nodes (ILNs) were stained for surface markers CD3 (1452C11), CD4 (RM4-5), CD25 (PC61); fixed/permeabilised with the Cytofix/Cytoperm kit (Thermo Fisher Scientific, Waltham, MA, USA); and additionally stained with anti-
FoxP3 monoclonal antibodies (NRRF-30) or IL-10 (JES516E3) monoclonal antibodies. For detection of intracellular IL-10, cells were stimulated in vitro for $3 \mathrm{~h}$ with a mixture of phorbol myristate acetate $(25 \mathrm{ng} / \mathrm{ml})$ and ionomycin $(1 \mu \mathrm{g} / \mathrm{ml})$ at $37^{\circ} \mathrm{C}, 5 \% \mathrm{CO}_{2}$ in the presence of Golgi Stop (Cytofix/Cytoperm kit, Thermo Fisher Scientific). All monoclonal antibodies were from Thermo Fisher Scientific. Cell data were acquired by LSR II flow cytometer (BD Bioscience, San Diego, CA, USA) and analysed using FlowJo software (Tree Star, Ashland, OR, USA).

\section{Results}

Diabetes incidence in bacteriome-humanised NOD mice The transfer of human bacteriome was associated with significantly lower diabetes incidence (logrank test $p_{\text {corrected }}$ from $2 \times 10^{-4}$ to 
0.025) in all of the bacteriome-humanised mice colonies as compared with the control colony of germ-free NOD mice that reached a $100 \%$ cumulative incidence on day 184 (Fig. 1a). Bacteriome-humanised colonies did not significantly differ from each other in diabetes-free survival rate (all corrected $p>0.1$ ),
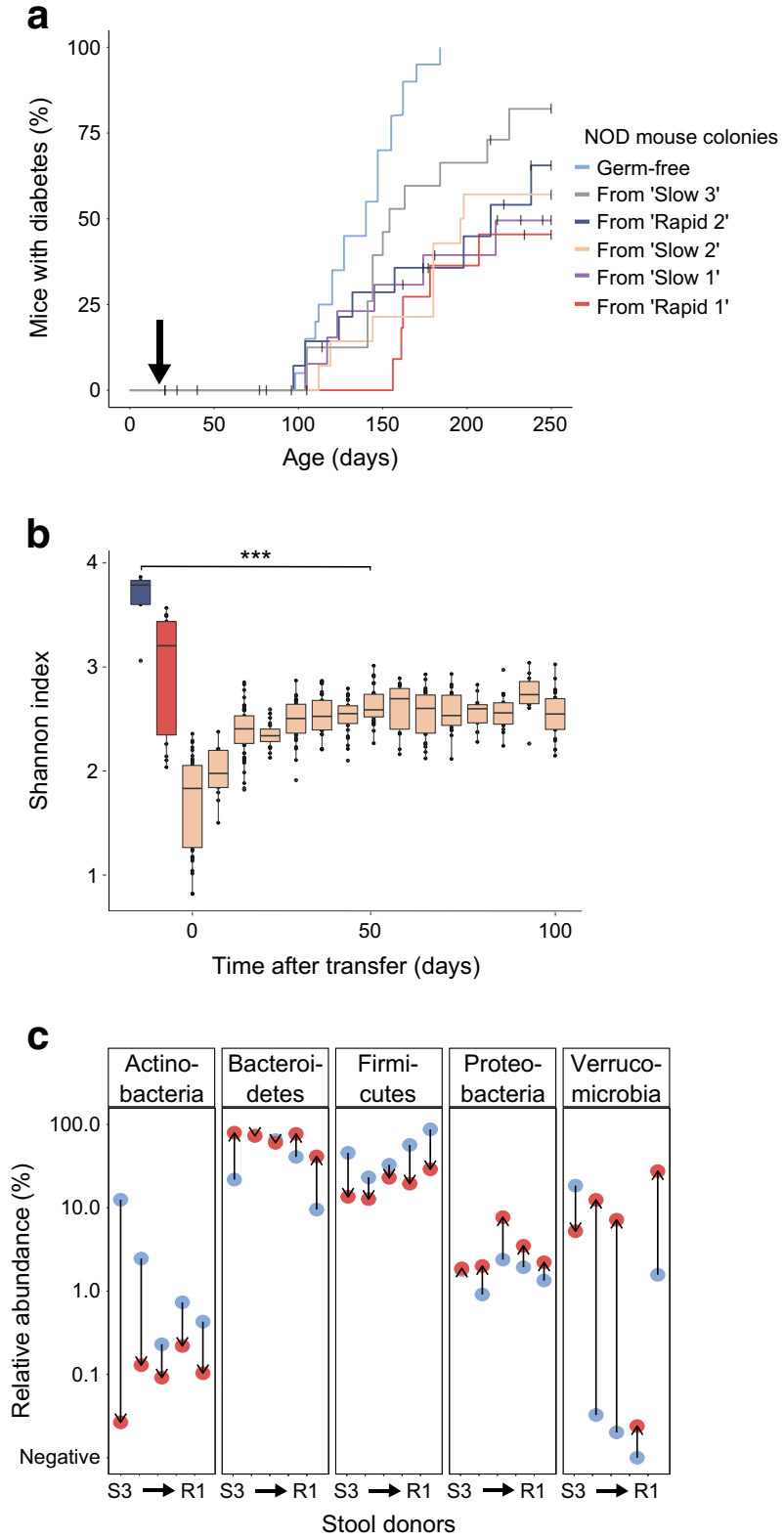

Fig. 1 NOD mice after human microbiota transfer. (a) The cumulative incidence of diabetes in mouse colonies after the stool transfer and in the control colony of germ-free mice. The time of the transfer is marked by the black arrow. (b) Shannon index of alpha diversity during and after the transfer process. Donor samples are in blue, the transferred material in red and mouse stools at predefined time-points in beige. (c) Comparison of the relative abundance (plotted on a log scale) of phyla in the donor (blue) and mouse stools (red) among donors and their respective colonies. Mouse data were analysed from stools taken at 3-8 weeks after the transfer. The donors are ordered from the mouse colony with the highest diabetes incidence (Slow 3 [S3]) to that with the lowest diabetes incidence (Rapid 1 [R1]). Control (germ-free), $n=20$; Rapid 1, $n=15$; Rapid 2, $n=16$; Slow $1, n=15$; Slow $2, n=18$; Slow $3, n=16$ regardless of donors' pace of beta cell function loss. The only pairwise difference approaching statistical significance was noted between the highest-incidence colony (transferred with microbiota from donor 'Slow 3') and the lowest-incidence colony (colonised from a donor with a rapid beta cell loss, 'Rapid 1'): we observed a 50-day lag between the times when the mice started to develop diabetes, and a difference of $36 \%$ in the cumulative diabetes incidence at day 250 post-transfer (logrank test $p_{\text {uncorrected }}=0.042, p_{\text {corrected }}=0.11$ ).

Gut bacteriome composition As compared with the donor stool and the inoculation mixture, the stool samples from mice had significantly lower alpha diversity $\left(p<10^{-5}\right.$ in all diversity indices). This drop in diversity following the transfer was especially prominent in early post-transfer samples and only partly restored during the first month thereafter (Fig. 1b). Bacteriome profiling from the transferred material yielded similar alpha diversity levels to the donor sample (Shannon index paired $t$ test $p=0.08$ ), which suggests that the loss occurred predominantly in the mouse gut.

The comparison of main bacterial phyla between the transferred stool material and the stabilised mouse bacteriomes (after 3-8 weeks post-transfer) showed a massive enrichment of the Bacteroidetes phylum to the detriment of the Firmicutes phylum $\left(\log _{e}\right.$ of the Firmicutes/Bacteroidetes ratio in donor vs mouse samples, paired $t$ test $p=0.0033$ ) (Fig. $1 \mathrm{c}$ and electronic supplementary material [ESM] Figs. 1, 2). The Actinobacteria phylum was also markedly decreased, especially in the highest-incidence colony. The changes in Verrucomicrobia were gross but not consistent between colonies.

At the genus level, some taxa frequent in the human gut were almost completely eliminated in all mouse colonies, e.g. Escherichia coli of the Proteobacteria or Bifidobacterium of the Actinobacteria phylum. Among the prevalent Bacteroidetes phylum, the changes upon transfer were many-fold and prominent, but no pattern could be observed among the mouse colonies (ESM Fig. 3 ). The genus Bacteroides increased in all but the lowestincidence colony, where it became nearly extinct, as was also confirmed by specific real-time PCR. The real-time PCR quantification of Bacteroides dorei showed its presence in one mouse colony (intermediate incidence level), whereas Bacteroides vulgatus was prevalent in all but the above-mentioned lowest-incidence colony (data not shown).

There was no bacteriome composition pattern associated with diabetes incidence in the mouse colonies - neither at the individual level nor at the colony level. The bacteriome composition of donor samples did not differ significantly by the assigned diets, and no systematic difference could be detected between bacteriomes from the slow and rapidly progressing human donors (data not shown). 
Insulitis and immune cell variables The germ-free mice scored significantly higher on the insulitis scores as compared with the bacteriome-humanised mouse colonies $(p<0.01$ for the tested bacteriome-humanised mice, ESM Fig. 4).

The proportions of FoxP $3^{+} \mathrm{CD} 25^{+} \mathrm{CD} 4^{+}$Tregs did not correspond to any specific pattern, e.g. mucosal (MLN, PLN) vs non-mucosal (spleen, ILN) compartments of 'Slow' vs 'Rapid' individuals. Except for MLNs from 'Rapid 2' colony, the bacteriome-humanised mice displayed similar or increased proportion of FoxP $3^{+} \mathrm{CD} 25^{+} \mathrm{CD} 4^{+}$Tregs (ESM Fig. 5). Except for spleens where statistically significant increased proportion of IL-10 $0^{+} \mathrm{T}$ helper cells was detected in human bacteriome-colonised mice $(p=0.009)$, no substantial differences were found in MLNs, PLNs and ILNs (ESM Fig. 6).

\section{Discussion}

Our results indicate that the phenotype of beta cell loss after the onset of type 1 diabetes is not transferable by gut microbiota into the germ-free NOD mouse model - therein the incidence of diabetes was lowered by any transferred microbiota, regardless of the donor phenotype. The pace of beta cell loss in the mouse model was independent of that in the human donor, as the highest-incidence mouse group was colonised from a child with slow beta cell function loss and vice versa. The mouse insulitis scoring was in line with the incidence data: the germ-free mice scored significantly higher than any of the bacteriome-humanised groups. The lack of an obvious difference in FoxP3 Treg counts between colonies and the increase of IL- $10^{+} \mathrm{T}$ helper cells in spleen corroborate previously published data suggesting lower or unchanged proportions of FoxP3 Tregs and IL- $10^{+} \mathrm{T}$ helper cells under germfree conditions [9-11]. Indeed, these immunological observations could be more conclusive if obtained from larger-sized groups and expressed as total immune cell numbers.

Our study offers insights into the microbial dynamics after the human microbiome transfer owing to the frequent stool collection from individual mice every 14 days. Despite the thorough colonisation approach (oral, rectal, dermal) with an adequate inoculum size, the human microbiome patterns were reproduced only imperfectly. Very dynamic changes were observed in the adopted microbiome and the microbiome stabilised only shortly before the first mice started to develop diabetes. A massive shift in the relative abundances of the main phyla was consistently observed in all colonies of bacteriome-humanised NOD mice, which renders further support to earlier smaller datasets from non-diabetic mice $[12,13]$. Several phyla seem to be unable to take root in the mouse gut as exemplified by the decrease of Actinobacteria in all mouse colonies.

We noted a marked drop in alpha diversity indices followed by stabilisation. This stabilisation took 4-5 weeks after human microbiota transfer. This is in contrast to the transfer of microbiota originating from mouse, which has been reported to be accepted by germ-free mice quicker and much more accurately [14]. Such a difference suggests that human microbiota must undergo a complex process of re-establishment in the mouse gut, probably passing through a certain kind of selection bottleneck. Once established, the resulting microbiome then remained very homogenous and stable over time without appreciable changes in mice who were about to develop diabetes. This stability can be also partly attributed to co-housing of the mice within the colony [15].

Although no systematic differences could be defined that would predict diabetes incidence in the bacteriomehumanised mice, we noted two candidate taxa having, maybe incidentally, an unusual distribution. First, the proportion of phylum Verrucomicrobia (i.e. its genus Akkermansia) decreased in the highest-incidence colony only, a fact noteworthy in connection to its mucin-degrading, gut protective properties [16]. Second, the genus Bacteroides was nearly absent in the lowest-incidence colony, being substituted by other two genera of Bacteroidetes, Alistipes and Dysgonomonas.

Our report provides new insights into the non-selective transfer of human stool to germ-free NOD mice. While previous research in NOD mice focused on selected probiotic [17] or pathogenic [18] bacteria, our approach allowed for full microbial interplay within the mouse gut. We observed large variability and inconsistency with which the germ-free NOD mice amplified some taxa upon the transfer from human donors, whereas other taxa were nearly eliminated. This, together with the dynamics of alpha diversity, suggests that the process is far too complex to render a reproducible model for assessing the net effects of human bacteriome on islet autoimmunity. Wos-Oxley et al demonstrated that the human gut bacteriome is reproduced more accurately by rats than mice [12]. Rather unfortunately, the immunopathology of islet autoimmunity in the available type 1 diabetes rat model, the BioBreeding $(\mathrm{BB})$ rat, has been demonstrated to profoundly differ from that in humans [19].

We clearly demonstrated that the pace of beta cell loss is not a trait readily transferable to NOD mice via the gut microbiota. This finding may have implications for the planning and design of novel human intervention trials.

Acknowledgements The content of this paper was presented at the annual conferences of International Society for Pediatric and Adolescent Diabetes (ISPAD) 2017 and Immunology of Diabetes (IDS) 2018. Some data were presented as an abstract at the ISPAD meeting in 2017.

Data availability All data used for the analysis in this article are available on request from the authors.

Funding This study received funding from the Ministry of Health of the Czech Republic (AZV grant 16-27994A).

Duality of interest The authors declare that there is no duality of interest associated with this manuscript. 
Contribution statement $\mathrm{VN}$ acquired and analysed the data and drafted the manuscript. LK and JG participated in the data analysis and critically revised the article. TH, LP and SP helped with the data acquisition and critically revised the manuscript. DF, ZS and OC have substantially contributed to the design of the study and revised the article. All co-authors were given the final version of the manuscript and approved its content. OC is the guarantor of this work and, as such, had full access to all of the data in the study and takes responsibility for the integrity of the data and the accuracy of the data analysis.

\section{References}

1. Knip M, Honkanen J (2017) Modulation of type 1 diabetes risk by the intestinal microbiome. Curr Diab Rep 17(11):105. https://doi. org/10.1007/s11892-017-0933-9

2. Davis-Richardson AG, Ardissone AN, Dias R et al (2014) Bacteroides dorei dominates gut microbiome prior to autoimmunity in Finnish children at high risk for type 1 diabetes. Front Microbiol 5:678

3. de Goffau MC, Fuentes S, van den Bogert B et al (2014) Aberrant gut microbiota composition at the onset of type 1 diabetes in young children. Diabetologia 57(8):1569-1577. https://doi.org/10.1007/ s00125-014-3274-0

4. Ludvigsson J, Carlsson A, Deli A et al (2013) Decline of C-peptide during the first year after diagnosis of Type 1 diabetes in children and adolescents. Diabetes Res Clin Pract 100(2):203-209. https:// doi.org/10.1016/j.diabres.2013.03.003

5. Tlaskalova-Hogenova H, Stepankova R, Kozakova H et al (2011) The role of gut microbiota (commensal bacteria) and the mucosal barrier in the pathogenesis of inflammatory and autoimmune diseases and cancer: contribution of germ-free and gnotobiotic animal models of human diseases. Cell Mol Immunol 8(2):110-120. https://doi.org/10.1038/cmi.2010.67

6. Hamilton MJ, Weingarden AR, Sadowsky MJ, Khoruts A (2012) Standardized frozen preparation for transplantation of fecal microbiota for recurrent Clostridium difficile infection. Am J Gastroenterol 107(5):761-767. https://doi.org/10.1038/ajg.2011. 482

7. Kozich JJ, Westcott SL, Baxter NT, Highlander SK, Schloss PD (2013) Development of a dual-index sequencing strategy and curation pipeline for analyzing amplicon sequence data on the MiSeq Illumina sequencing platform. Appl Environ Microbiol 79(17):5112-5120. https://doi.org/10.1128/AEM.01043-13

8. Cinek O, Kramna L, Lin J et al (2017) Imbalance of bacteriome profiles within the Finnish Diabetes Prediction and Prevention study: parallel use of $16 \mathrm{~S}$ profiling and virome sequencing in stool samples from children with islet autoimmunity and matched controls. Pediatr Diabetes 18(7):588-598. https://doi.org/10.1111/pedi. 12468
9. Alam C, Bittoun E, Bhagwat D et al (2011) Effects of a germ-free environment on gut immune regulation and diabetes progression in non-obese diabetic (NOD) mice. Diabetologia 54(6):1398-1406. https://doi.org/10.1007/s00125-011-2097-5

10. Atarashi K, Tanoue T, Oshima K et al (2013) Treg induction by a rationally selected mixture of Clostridia strains from the human microbiota. Nature 500(7461):232-236. https://doi.org/10.1038/ nature12331

11. Atarashi K, Tanoue T, Shima T et al (2011) Induction of colonic regulatory $\mathrm{T}$ cells by indigenous Clostridium species. Science 331(6015):337-341. https://doi.org/10.1126/science.1198469

12. Wos-Oxley M, Bleich A, Oxley AP et al (2012) Comparative evaluation of establishing a human gut microbial community within rodent models. Gut Microbes 3(3):234-249. https://doi.org/10. 4161/gmic. 19934

13. Turnbaugh PJ, Ridaura VK, Faith JJ, Rey FE, Knight R, Gordon JI (2009) The effect of diet on the human gut microbiome: a metagenomic analysis in humanized gnotobiotic mice. Sci Transl Med 1:6-14

14. El Aidy S, van Baarlen P, Derrien M et al (2012) Temporal and spatial interplay of microbiota and intestinal mucosa drive establishment of immune homeostasis in conventionalized mice. Mucosal Immunol 5(5):567-579. https://doi.org/10.1038/mi.2012. 32

15. Zhang L, Bahl MI, Roager HM et al (2017) Environmental spread of microbes impacts the development of metabolic phenotypes in mice transplanted with microbial communities from humans. ISME J 11(3):676-690. https://doi.org/10.1038/ismej.2016.151

16. Hanninen A, Toivonen R, Poysti S et al (2017) Akkermansia muciniphila induces gut microbiota remodelling and controls islet autoimmunity in NOD mice. Gut 67:1445-1453

17. Calcinaro F, Dionisi S, Marinaro M et al (2005) Oral probiotic administration induces interleukin-10 production and prevents spontaneous autoimmune diabetes in the non-obese diabetic mouse. Diabetologia 48(8):1565-1575. https://doi.org/10.1007/s00125005-1831-2

18. Lee AS, Gibson DL, Zhang Y, Sham HP, Vallance BA, Dutz JP (2010) Gut barrier disruption by an enteric bacterial pathogen accelerates insulitis in NOD mice. Diabetologia 53(4):741-748. https://doi.org/10.1007/s00125-009-1626-y

19. Mordes JP, Bortell R, Blankenhorn EP, Rossini AA, Greiner DL (2004) Rat models of type 1 diabetes: genetics, environment, and autoimmunity. ILAR J 45(3):278-291. https://doi.org/10.1093/ilar. 45.3.278

Publisher's note Springer Nature remains neutral with regard to jurisdictional claims in published maps and institutional affiliations. 